\title{
THE MOTIONS OF ALGEBRAIC DIFFERENTIAL EQUATIONS
}

\author{
by LEE A. RUBEL
}

(Received 30 September, 1982)

We confine ourselves, for simplicity, to first-order algebraic differential equations (ADE's), although analogous considerations may be made for higher-order ADE's:

$$
P\left(x, y(x), y^{\prime}(x)\right)=0 .
$$

A motion of $\left(^{*}\right)$ is a change of independent variable that takes solutions to solutions, that is, a suitable map $\varphi$ of the underlying interval $I$ into itself so that if $y$ is a solution of $\left(^{*}\right)$ then $y \circ \varphi$ is a solution of $\left(^{*}\right)$, i.e.

$$
P\left(x, y(\varphi(x)), y^{\prime}(\varphi(x)) \varphi^{\prime}(x)\right)=0 .
$$

In this paper we prove that the motions satisfy their own second order ADE

$$
Q\left(x, \varphi(x), \varphi^{\prime}(x), \varphi^{\prime \prime}(x)\right)=0
$$

and that, in general, (\#) cannot be replaced by a first-order ADE. We find this surprising.

THEOREM 1. Consider the equation $\left(^{*}\right)$ on an open interval $I \subseteq \mathbb{R}$. There exists an equation (\#) that is satisfied by every $C^{2}$ motion $\varphi$ of $\left(^{*}\right)$ for which there exists a $C^{1}$ solution $y$ for which $y \circ \varphi$ is not a constant on any subinterval of $I$.

It is clear that some restriction on the ADE is needed. Consider, for example the ADE $y^{\prime}=0$, where the solutions are the constant functions, and any mapping is a motion. [1]).

Before proving this, let us briefly review some classical things about resultants (see For

$$
\begin{gathered}
A(Y)=a_{0} Y^{a}+a_{1} Y^{q-1}+\ldots+a_{q}, \\
B(Y)=b_{0} Y^{r}+b_{1} Y^{r-1}+\ldots+b_{r},
\end{gathered}
$$

we define the $Y$-resultant of $A(Y)$ and $B(Y)$ by the formula

$$
\left.\operatorname{Res}_{Y}(A(Y), B(Y))=\operatorname{det}\left[\begin{array}{ccccccccccc}
a_{0} & a_{1} & & \ldots & & a_{\mathrm{q}} & 0 & & \ldots & \ldots & 0 \\
0 & a_{0} & a_{1} & & \ldots & & a_{\mathrm{q}} & 0 & \ldots & \ldots & 0 \\
\vdots & & & & & & & & & & \\
0 & & \ldots & & a_{0} & a_{1} & \ldots & \ldots & \ldots & a_{\mathrm{a}} \\
b_{0} & b_{1} & & \ldots & & b_{r} & 0 & \ldots & \ldots & \ldots & 0 \\
0 & b_{0} & b_{1} & & \ldots & & b_{r} & 0 & \ldots & \ldots & 0 \\
\vdots & & & & & & & & & & \\
0 & & \ldots & b_{0} & b_{1} & \ldots & \ldots & \ldots & \ldots & b_{r}
\end{array}\right]\right\} r \text { rows }
$$

Glasgow Math. J. 25 (1984) 93-96. 
This has the following property, where for simplicity we assume $a_{0}=1=b_{0}: A(Y)=0$ and $B(Y)=0$ have a common solution if and only if $\operatorname{Res}_{Y}(A(Y), B(Y))=0$.

Now let us prove Theorem 1. Substituting $\varphi(x)$ for $x$ in $\left(^{*}\right)$, we have

$$
P\left(\varphi(x), y(\varphi(x)), y^{\prime}(\varphi(x))\right)=0
$$

and

$$
P\left(x, y(\varphi(x)), y^{\prime}(\varphi(x)) \varphi^{\prime}(x)\right)=0
$$

because $\varphi$ is a motion. Considering the left hand sides of (1) and (2) as polynomials in $y^{\prime}(\varphi(x))$ we see, on factoring out the highest powers of $y^{\prime}(\varphi(x))$ permissible, that

$$
\begin{gathered}
\tilde{P}\left(\varphi(x), y(\varphi(x)), y^{\prime}(\varphi(x))=0,\right. \\
\tilde{P}\left(x, y(\varphi(x)), y^{\prime}(\varphi(x)) \varphi^{\prime}(x)\right)=0,
\end{gathered}
$$

where $\tilde{P}(x, y, 0) \neq 0$. (This is because of our hypothesis which implies that there exist solutions of $(1)$ and $(2)$ with $y^{\prime}(\varphi(x)) \neq 0$.)

Taking resultants of these two polynomials in $y^{\prime}(\varphi(x))$, we eliminate $y^{\prime}(\varphi(x))$ to get

$$
S\left(x, \varphi(x), \varphi^{\prime}(x), y(\varphi(x))\right)=0 .
$$

Now differentiate this expression to get

$$
T\left(x, \varphi(x), \varphi^{\prime}(x), \varphi^{\prime \prime}(x), y\left(\varphi(x), y^{\prime}(\varphi(x))\right)=0 .\right.
$$

This time, use resultants to eliminate $y^{\prime}(\varphi(x))$ between (1) and (4) to get

$$
U\left(x, \varphi(x), \varphi^{\prime}(x), \varphi^{\prime \prime}(x), y(\varphi(x))\right)=0 .
$$

Now we may divide (3) and (5) by the highest permissible powers of $y(\varphi(x))$ to get

$$
\begin{gathered}
\tilde{S}\left(x, \varphi(x), \varphi^{\prime}(x), y(\varphi(x))\right)=0, \\
\tilde{U}\left(x, \varphi(x), \varphi^{\prime}(x), \varphi^{\prime \prime}(x), y(\varphi(x))\right)=0,
\end{gathered}
$$

where $\tilde{\boldsymbol{S}}(x, y, z, 0) \neq 0$ and where $\tilde{U}(x, y, z, w, 0) \neq 0$. This is possible since (3) and (5) have solutions where $y(\varphi(x))$ is not identically zero on. any subinterval of $I$. Again taking a resultant, we eliminate $y(\varphi(x))$ from $\left(3^{\prime}\right)$ and $\left(5^{\prime}\right)$ to get

$$
Q\left(x, \varphi(x), \varphi^{\prime}(x), \varphi^{\prime \prime}(x)\right)=0
$$

which is the desired result.

THEOREM 2. In the context of the above theorem, one may not generally take $\varphi$ to satisfy a first-order algebraic differential equation.

Proof. Let the given ADE be

$$
y^{\prime}+2 x y=1
$$

whose general solution is

$$
y=e^{-x^{2}} \int_{0}^{x} e^{t^{2}} d t+d e^{-x^{2}}, d \text { a constant }
$$


If $\varphi$ is a motion of $\left(^{*}\right)$ then

$$
\begin{gathered}
e^{-\varphi^{2}} \int_{0}^{\varphi} e^{t^{2}} d t=e^{-x^{2}} \int e^{t^{2}} d t+c e^{-x^{2}} \\
c=c(\varphi)=\text { const. }
\end{gathered}
$$

Take the derivative of this last expression

$$
e^{x^{2}-\varphi^{2}}\left(2 x-2 \varphi \varphi^{\prime}\right) \int_{0}^{\varphi} e^{t^{2}} d t+e^{x^{2}-\varphi^{2}} e^{\varphi^{2}} \varphi^{\prime}-e^{x^{2}}=0
$$

which we write as

$$
\varphi^{\prime}=\frac{1-2 x \operatorname{err} \varphi}{1-2 \varphi \operatorname{err} \varphi}
$$

where

$$
\operatorname{err} \varphi=e^{-\varphi^{2}} \int_{0}^{\varphi} e^{t^{2}} d t
$$

Now (6) is a first-order differential equation, but it is not algebraic. Now over a rectangle $R$ in the $(x, z)$ plane, there is a unique solution to (6) with $\varphi\left(x_{0}\right)=z_{0}$ for $\left(x_{0}, z_{0}\right) \in R$. Thus for $\left(x_{0}, z_{0}\right) \in R$, we would have

if we were to have

$$
Q\left(x_{0}, z_{0},\left(\frac{1-z x_{0} \text { err } z_{0}}{1-2 z_{0} \text { err } z_{0}}\right)\right)=0
$$

$$
Q\left(x, \varphi(x), \varphi^{\prime}(x)\right)=0
$$

for some polynomial $Q$ in three variables. We will show that this is impossible. Let us rewrite this as

$$
Q\left(x, z, \frac{1-2 x \operatorname{err} z}{1-2 z \operatorname{err} z}\right)=0 \quad \text { for } \quad(x, z) \in R .
$$

Hold $x=x_{0}$ fixed in $R$. Then for an interval $I$ of values of $z$

$$
Q\left(x_{0}, z, \frac{1-2 x_{0} \operatorname{err} z}{1-2 z \text { err } z}\right)=0
$$

Now (7) implies, unless $Q\left(x_{0}, z, w\right) \equiv 0$, that err $z$ is an algebraic function of $z$ over $I$. However from [2, pp. 48-49] this is not so. The only way to resolve this contradiction is that

$$
Q\left(x_{0}, z, w\right) \equiv 0 \text { for }\left(x_{0}, z\right) \text { in } R, w \in \mathbb{C} \text {. }
$$

This implies $Q(x, y, z) \equiv 0$, and thus we have our conclusion that there is no non-trivial first-order ADE satisfied by all the motions $\varphi$. 


\section{REFERENCES}

1. W. S. Burnside and A. W. Panton, Theory of equations (Dublin, 1904). (Also Dover reprint.)

2. J. F. Ritt, Integration in finite terms (Columbia U. P., 1948).

Department of Mathematics

UNIVERSITY OF ILLINOIS

URBANA

ILLLINOIS 61801

U.S.A. 